\title{
Prevalência de Síndrome Metabólica em Estudo de Base Populacional, Vitória, ES - Brasil
}

\begin{abstract}
RESUMO
Síndrome Metabólica (SM) é um transtorno representado pela agregação de fatores predisponentes para desenvolvimento de doenças cardiovasculares e diabetes. Apesar da importância da SM, há carência de dados sobre as características epidemiológicas desta condição na população brasileira. Determinamos a prevalência da SM por sexo, faixa etária e nível socioeconômico na população da cidade de Vitória, ES, Brasil, utilizando os critérios do NCEP/ATPIII. Foram coletados dados socioeconômicos, bioquímicos, antropométricos e hemodinâmicos em 1.663 indivíduos de amostra randômica da população (25-64 anos) de Vitória. A prevalência foi de $29,8 \%$ (IC95 = 28-32\%), sem diferença entre sexos. De 25 a 34 anos, a prevalência foi $15,8 \%$, alcançando $48,3 \%$ na faixa de 55 a 64 anos. Verificouse aumento progressivo de prevalência em mulheres do maior para o menor nível socioeconômico. O parâmetro da SM mais freqüente em homens foi hipertensão, seguido de hipertrigliceridemia, baixo HDL-colesterol, hiperglicemia e obesidade abdominal. Nas mulheres, hipertensão em primeiro lugar, seguida do baixo HDLcolesterol, obesidade abdominal, hipertrigliceridemia e hiperglicemia. Conclui-se que a prevalência de SM é elevada, inclusive nos mais jovens, com grande contribuição da hipertensão para o seu diagnóstico. Controle dos fatores de risco deve ser promovido visando reduzir o impacto das doenças cardiovasculares na mortalidade geral. (Arq Bras Endocrinol Metab 2007;51/7:1143-1152)
\end{abstract}

Descritores: Síndrome metabólica; Hipertensão; Resistência à insulina; Obesidade abdominal

\section{ABSTRACT}

Prevalence of Metabolic Syndrome in Population-Based Study, Vitória, ES - Brazil.

Metabolic Syndrome (MS) is a complex disorder including several factors predisposing to development of cardiovascular diseases and diabetes. Despite the importance of MS for the health system, the epidemiological characteristics of this condition in the Brazilian population are still scarce. The prevalence of MS as a function of gender, age and socioeconomic level was determined in a population-based study in Vitória, ES, Brazil, by using the NCEP-ATPIII diagnosis criteria. Socioeconomic, biochemical, anthropometric, and hemodynamic data were obtained in 1,663 individuals from a random sample of Vitória population (25-64 y). The estimated prevalence of MS was $29,8 \%$ (CI95 = 28-32\%). No significant sex-related differences were observed. Prevalence increased from the youngest $(26-34 \mathrm{y})$ to the oldest (55-64 y) group ( $15.8 \%$ and $48.3 \%$, respectively). A progressive increase of MS frequency was observed in women from the higher to the lowest socioeconomic level. The most frequent trait of MS in males was high blood pressure, followed by hypertriglyceridemia, low HDL-c levels, hyperglycemia, and central obesity. In females, hypertension was also the most frequent factor, followed by low HDL-c levels, abdominal obesity, hypertriglyceridemia and hyperglycemia. Our data show that prevalence of MS is high in the studied population, even in the youngest group. Moreover, high blood pressure gives a significant contribution to the diagnosis of this syndrome in both sexes. The precocious control of risk factors is necessary to reduce the impact of cardiovascular morbidity and mortality. (Arq Bras Endocrinol Metab 2007;51/7:1143-1152)

Keywords: Metabolic syndrome; Hypertension; Insulin resistance; Abdominal obesity artigo original

\author{
LUCIANE B. SALAROLI \\ GeOVANE C. BARBOSA \\ JoSÉ G. MILL \\ MARIA C.B. MOLINA
}

Programa de Pós-Graduação em Atenção à Saúde Coletiva e Programa de Pós-Graduação em Ciências Fisiológicas - UFES, Vitória, ES. Aceito em 14/05/07 
A s STRANSFORMAÇÕES OCORRIDAS nOS padrões sOcioeconômicos e culturais da população a partir da segunda metade do século XX alteraram de forma significativa o modo de vida da humanidade. Se, por um lado, houve avanços importantes que contribuíram para aumentar e melhorar a vida do homem, por outro incrementaram mudanças marcantes que influem no processo saúde-doença (1), tais como as alterações nos hábitos alimentares e no gasto energético relacionado às atividades diárias e atividade física.

Além dessas, o estresse causado pela vida moderna e urbana provavelmente tem contribuído sobremaneira para o aumento da incidência de várias doenças crônicas, tais como a obesidade, o diabetes mellitus e a hipertensão arterial, as quais freqüentemente cursam com alterações nas lipoproteínas plasmáticas e aumento de risco para as doenças cardiovasculares (2). A simultaneidade dessas alterações, aliada a um quadro de resistência à insulina, compõe a chamada síndrome metabólica (SM) (3).

A SM representa a anormalidade metabólica mais comum da atualidade e também a maior responsável por eventos cardiovasculares na população (3). O desenvolvimento da SM em determinado indivíduo depende de uma complexa interação entre a predisposição genética e fatores ligados ao estilo de vida, como padrão dietético, sedentarismo e obesidade, o que caracteriza a natureza multifatorial da patogênese da SM (4).

Reaven (1988) identificou a resistência à insulina, definida como a menor captação de glicose pelos tecidos periféricos (5), e denominou este quadro, originalmente, como a "Síndrome X". Atualmente, de forma mais ampla, a SM tem sido caracterizada pela presença concomitante de dislipidemia, distúrbio da tolerância à glicose, hipertensão arterial, excesso de peso ou obesidade abdominal, além de outras anormalidades. Aparentemente, a resistência à insulina parece ser o elo de ligação entre tais achados (3), a qual está vinculada de uma forma não totalmente conhecida ao aumento da deposição de gordura visceral. A presença de SM é forte preditor de mortalidade cardiovascular (6). Dessa forma, deve-se destacar sua importância do ponto de vista epidemiológico, tendo em vista a elevada mortalidade cardiovascular presente em todos os países desenvolvidos e em países em desenvolvimento, como o Brasil (3). Em estudo retrospectivo, Lakka e cols. (7) verificaram que a mortalidade coronariana, cardiovascular e por todas as causas em homens de 42 a 60 anos de idade, e inicialmente sem doença cardiovascular (DCV), câncer e diabetes, foi maior nos indivíduos com SM.
No Brasil, os resultados da Pesquisa Nacional de Saúde e Nutrição (PNSN), realizada em 1989, já evidenciavam um aumento da obesidade e redução dos índices de desnutrição, particularmente em regiões economicamente mais desenvolvidas (8). Dados da última Pesquisa de Orçamento Familiar (POF), realizada pelo IBGE em 2002/03, confirmaram essa tendência, com a obesidade acometendo $8,9 \%$ dos homens e $13,1 \%$ das mulheres (9). Esse fato merece destaque já que o excesso de peso, localizado principalmente na região abdominal, está diretamente associado às alterações no perfil lipídico, ao aumento da pressão arterial e à hiperinsulinemia, fatores esses que aumentam o risco do diabetes tipo 2 e das DCV (10).

Em estudo realizado na Holanda (11), a SM estava presente em $58 \%$ dos indivíduos com doença vascular periférica, em $41 \%$ daqueles com doença arterial coronariana, em $43 \%$ daqueles com doenças cerebrovasculares e em $47 \%$ dos portadores de aneurisma da aorta abdominal.

O Brasil apresenta um quadro preocupante em relação às $\mathrm{DCV}$, não só pelas elevadas taxas de morbimortalidade, mas principalmente por elas estarem afetando de forma importante os estratos etários mais jovens $(6,12)$. O aparecimento precoce da SM em nossa população, que sabidamente apresenta níveis elevados e crescentes de obesidade (9), poderia estar relacionado a esse quadro, cuja mortalidade por infarto do miocárdio em homens com menos de 55 anos chega a ser 3 a 4 vezes maior do que em países desenvolvidos (13). Apesar da importância da SM no contexto das doenças metabólicas e cardiovasculares (7), tanto a prevalência como as demais características epidemiológicas da SM ainda são pouco conhecidas em nossa população. Assim, este trabalho teve por objetivo determinar a prevalência de SM na cidade de Vitória e verificar quais componentes são mais importantes associados à sua presença em população do Estado do Espírito Santo, Brasil.

\section{MATERIAL E MÉTODOS}

Foi realizado estudo observacional, transversal, cuja base populacional foi constituída pelos moradores do Município de Vitória, Espírito Santo, Brasil, com idades de 25 a 64 anos, que fizeram parte de um estudo para determinar prevalência e gravidade dos fatores de risco cardiovascular (Projeto MONICA-OMS/Vitória). Os dados foram coletados de abril de 1999 a novembro de 2000. O estudo foi aprovado pelo Comitê de Ética em Pesquisa do Centro de Ciências da Saúde da Universidade Federal do Espírito Santo. 
Foram selecionados 2.268 moradores de Vitória a partir de uma amostra aleatória de igual número de domicílios. A amostragem foi programada de modo a cobrir proporcionalmente todas as 7 regiões administrativas da cidade de Vitória (14). Foram inicialmente sorteados bairros e, dentro destes, setores censitários. O sorteio dos domicílios dentro de cada setor foi feito por mecanismo de aleatorização. Caso não houvesse nenhum morador no domicílio sorteado, era selecionado o domicílio imediatamente seguinte, até se completar a cota de indivíduos de cada setor censitário. Em cada domicílio foi sorteado apenas um indivíduo, situado na faixa etária do estudo, para fazer parte da pesquisa. Após a escolha do indivíduo (data de aniversário mais próxima à da visita), este era convidado a assinar o termo de consentimento livre e esclarecido para sua participação no estudo. A coleta de dados era iniciada no próprio domicílio por meio de levantamento de dados socioeconômicos e de saúde. A seguir, era agendado o dia para que o participante comparecesse à Clínica de Investigação Cardiovascular do Centro de Ciências da Saúde da UFES para realização de exames clínicos e laboratoriais necessários à determinação de presença de fatores de risco cardiovascular. Dos 2.268 indivíduos incluídos na pesquisa durante a visita domiciliar, $1.663(73,3 \%)$ compareceram ao local de exames para a coleta de sangue em jejum de 12 horas, medida da pressão arterial, registro do eletrocardiograma e obtenção de medidas antropométricas, além de outros dados incluídos no protocolo de pesquisa. A classe socioeconômica foi determinada a partir da escolaridade do chefe da família e do número e diversidade de bens de consumo duráveis disponíveis no domicílio, sendo os indivíduos divididos em 5 classes, da mais alta (classe A) até a mais baixa (classe E). A classificação étnico-racial de cada indivíduo foi feita a partir da análise de características fenotípicas e informações do próprio indivíduo sobre os ancestrais. Os indivíduos foram divididos em 4 grupos: brancos, negros, mulatos e outros mestiços. Foram classificados como mulatos os indivíduos mestiços com uma ascendência negra predominante em vista dos dados fenotípicos. No último grupo foram incluídos mestiços não enquadráveis na categoria de mulato. Um pequeno número de indivíduos foi enquadrado nas categorias de asiático (4) ou indígena (7).

A pressão arterial foi medida no braço não dominante com esfigmomanômetro de mercúrio, com precisão de 2 $\mathrm{mmHg}$, com o indivíduo sentado e em repouso de, pelo menos, 5 minutos e após esvaziamento vesical. Duas medidas foram feitas por dois investigadores diferentes, previamente treinados, e com intervalo de cerca de 30 minutos entre as medidas. A pressão arterial de cada indivíduo foi obtida pela média das duas medidas. Braçadeira apropriada para obesos foi usada quando necessário. Todas as medidas antropométricas foram feitas por um único pesquisador ao longo de todo o projeto. O peso corporal foi obtido em balança eletrônica (precisão de $0,1 \mathrm{~kg}$ ) com a bexiga vazia e com o indivíduo portando apenas roupas íntimas. A estatura foi obtida em estadiômetro de parede com o indivíduo descalço e com precisão de $0,5 \mathrm{~cm}$. Desta forma, calculou-se o índice de massa corporal $\left(\mathrm{IMC}=\right.$ Peso $/$ Estatura $\left.^{2}\right)$, conforme recomendação da Organização Mundial de Saúde para avaliação do estado nutricional (15). Foram utilizados os seguintes pontos de corte para a classificação dos indivíduos quanto ao IMC $\left(\mathrm{kg} / \mathrm{m}^{2}\right)$ : baixo peso (IMC $\left.<18\right)$; normal $\geq$ 18,5 e $<25$; pré-obesidade $\geq 25$ e $<30$ e obesidade $\geq 30$. As obesidades de grau I, II e III foram agrupadas para tornar o grupo mais representativo. Foram obtidas as circunferências da cintura (CC) e do quadril (CQ) com fita métrica metálica. Para a medida da CC, a fita era posicionada na cintura natural ou na menor curvatura localizada entre o último arco costal e a crista ilíaca. A CQ foi medida na área de maior protuberância posterior dos glúteos.

Os exames bioquímicos foram feitos em um único local (Laboratório Central do Sesi/Vitória) com kits comerciais, tendo sido medidos os níveis de glicose, colesterol total, HDL-colesterol, VLDL-colesterol e triglicerídios. A concentração de LDL-colesterol foi calculada pela fórmula de Friedwald.

A SM foi identificada levando-se em consideração os parâmetros definidos pela Primeira Diretriz Brasileira de Diagnóstico e Tratamento da Síndrome Metabólica (3), a qual se baseia nos critérios definidos pelo National Cholesterol Education Program's - Adult Treatment Panel III (NCEP - ATP III). Segundo o NCEP - ATP III, a SM representa a combinação de, pelo menos, três dos cinco parâmetros usados para definir a síndrome: circunferência abdominal elevada ( $>102 \mathrm{~cm}$ para homens e $>88 \mathrm{~cm}$ para mulheres), aumento de triglicerídeos $(\geq 150 \mathrm{mg} / \mathrm{dL}$ ), HDL-colesterol baixo $(<40 \mathrm{mg} / \mathrm{dL}$ para homens $\mathrm{e}<50$ $\mathrm{mg} / \mathrm{dL}$ para mulheres), glicemia de jejum elevada $(\geq 110$ $\mathrm{mg} / \mathrm{dL}$ ) ou presença de diabetes, e aumento da pressão arterial (pressão sistólica $\geq 130 \mathrm{mmHg}$ e/ou pressão diastólica $\geq 85 \mathrm{mmHg}$, ou uso de anti-hipertensivos).

As análises estatísticas foram realizadas através da utilização do programa SPSS for Windows, versão 10.0.1 (1999). As médias foram comparadas utilizando-se o teste $t$ de Student para amostras independentes. Para análise das diferenças das proporções foi utilizado o teste do quiquadrado $\left(\chi^{2}\right)$. O nível de significância para todos os testes foi estabelecido em $\alpha=0,05$. Para as variáveis que não apresentaram uma distribuição normal, aplicou-se a transformação logarítmica (normalidade dos dados) para a realização do teste $\mathrm{t}$ para amostras independentes. Aplicouse nos dados de triglicerídeos a transformação logarítmica, sendo feita uma inspeção prévia de normalidade através do histograma e confirmada pelo teste de normalidade de Kolmogorov Smirnov com p valor $(0,133)$, indicando, assim, a aceitação da hipótese de normalidade dos dados.

\section{RESULTADOS}

Dos indivíduos selecionados nos domicílios, 1.663 indivíduos $(73,3 \%)$ compareceram às visitas agendadas na Clínica de Investigação Cardiovascular da UFES. 
Nos dados deste trabalho foram excluídos 33 indivíduos por não terem todos os exames necessários ao diagnóstico da SM. Portanto, os dados referem-se a 1.630 indivíduos, sendo $743(45,6 \%)$ homens e 887 mulheres $(54,4 \%)$, valores estes similares à distribuição por sexo na população de Vitória $(47,2$ e $52,8 \%$ ) na faixa etária do estudo, segundo os dados do censo de 2000 (16).

A tabela 1 mostra a distribuição percentual da amostra por sexo, faixa etária, grupo racial e escolaridade. Foram identificados 485 (29,8\%) indivíduos com SM, sendo 218 do sexo masculino e 267 do sexo feminino. A prevalência total da SM na amostra estudada foi de $29,8 \%$ (IC 95\% 28-32), sem haver diferença entre sexos. Nota-se incremento da prevalência da SM com o aumento da idade, tanto nos homens, quanto nas mulheres, não havendo diferença significativa entre sexos em nenhuma das faixas etárias do estudo. Chama atenção a prevalência elevada da SM mesmo na faixa etária mais baixa.

Um quadro interessante emerge ao analisar-se a prevalência da SM segundo a estratificação socioeconômica. Nesta análise, as classes D e E foram agrupadas em vista do pequeno número de indivíduos da classe $\mathrm{E}$. Ao se analisar a amostra global, observa-se tendência a aumento da prevalência da SM nos estratos socioeconômicos mais baixos (tabela 1). Esse aumento, entretanto, deveu-se exclusivamente à influência da classe na distribuição da SM encontrada no sexo feminino, onde a presença de mulheres com SM foi duas vezes maior nas classes $\mathrm{D}+\mathrm{E}(38,7 \%)$ do que na classe $\mathrm{A}$, a mais alta $(17,9 \%)$. O efeito classe socioeconômica na distribuição de prevalência da SM não parece ser decorrente de influência na escolaridade. Nos homens, a escolaridade não afetou a prevalência. Nas mulheres, apesar do efeito significante na escolaridade $(\mathrm{P}<0,05)$, não se observa linha de tendência regular da prevalência em função desse fator. Finalmente, a prevalência da SM foi similar entre os diferentes grupos étnico-raciais.

A tabela 2 mostra o estado nutricional de acordo com o IMC das mulheres com e sem SM, segundo a classe socioeconômica. Os dados revelam que as mulheres com SM das classes C e D apresentam maior freqüência de pré-obesidade e obesidade, quando comparadas com as sem SM.

A tabela 3 apresenta os indicadores bioquímicos, antropométricos e hemodinâmicos, por sexo, nos indivíduos com e sem SM. Entre os indivíduos sem a SM observa-se, como esperado, maiores valores de triglicerídeos, circunferência de cintura, RCQ e pressão arterial no sexo masculino em comparação ao feminino. Também como esperado, os níveis de HDL-c são significativamente maiores em mulheres. Ainda como esperado, os indicadores bioquímicos, antropométricos e pressóricos são maiores, dentro de cada sexo, nos portadores de SM, à exceção do HDL-c. Interessante observar que, para este último parâmetro, a diferença nas mulheres portadoras e não portadores da $S M$ $(41,3 \pm 8,2 \mathrm{mEq} / \mathrm{L}$ vs. $50,9 \pm 12,2 \mathrm{mEq} / \mathrm{L})$ é praticamente a mesma que nos portadores e não portadores de SM do sexo masculino $(35,9 \pm 7,3$ $\mathrm{mEq} / \mathrm{L}$ vs. $44,7 \pm 12,9 \mathrm{mEq} / \mathrm{L})$.

A figura 1 mostra a contribuição relativa dos cinco critérios para o diagnóstico da SM. Com relação aos parâmetros avaliados, os mais freqüentes entre os homens foram, seqüencialmente, a hipertensão arte-

Tabela 1. Prevalência de síndrome metabólica por sexo, faixa etária e grupo racial na amostra estudada em Vitória, ES, Brasil.

\begin{tabular}{|c|c|c|c|c|c|c|c|c|c|c|c|}
\hline Características & & Homens & & Valor de $p$ & & Mulheres & & Valor de p & & & Valor de p \\
\hline Idade (anos) & $\mathrm{N}$ & $\mathrm{P}$ & IC & $<0,00^{*}$ & $\mathrm{~N}$ & $P$ & IC & $0,00^{*}$ & $\mathrm{P}$ & IC & $<0,00^{*}$ \\
\hline & & $(\%)$ & (95\%) & & & $(\%)$ & (95\%) & & $(\%)$ & (95\%) & \\
\hline $25 \mid-34$ & 29 & 16,6 & $11-22$ & & 31 & 15,0 & $10-20$ & & 15,8 & $12-19$ & \\
\hline $35 \mid-44$ & 56 & 27,5 & $21-33$ & & 53 & 22,9 & $18-28$ & & 25,1 & $21-29$ & \\
\hline $45 \mid-54$ & 78 & 33,5 & $27-39$ & & 97 & 33,6 & 28-39 & & 33,5 & $29-38$ & \\
\hline $55 \mid-64$ & 55 & 42,0 & $33-50$ & & 86 & 53,4 & $46-61$ & & 48,3 & $43-54$ & \\
\hline Total & 218 & 29,3 & $23-35$ & & 267 & 30,1 & $24-36$ & & 29,8 & $28-32$ & \\
\hline $\begin{array}{l}\text { Classe } \\
\text { Socioeconômica }\end{array}$ & $\mathrm{N}$ & & & 0,48 & $\mathrm{~N}$ & & & $<0,00^{*}$ & & & $<0,05^{*}$ \\
\hline A & 24 & 29,6 & 19-39 & & 15 & 17,9 & $10-26$ & & 23,6 & $17-30$ & \\
\hline B & 65 & 32,2 & $25-38$ & & 50 & 20,7 & $16-26$ & & 25,9 & $22-30$ & \\
\hline C & 68 & 31,1 & $25-37$ & & 94 & 33,3 & 28-39 & & 32,3 & $28-36$ & \\
\hline$D$ e $E$ & 61 & 25,3 & $20-30$ & & 108 & 38,7 & $33-44$ & & 33,0 & $28-37$ & \\
\hline Grupo racial & $\mathrm{N}$ & & & 0,26 & $\mathrm{~N}$ & & & 0,07 & & & 0,36 \\
\hline Brancos & 84 & 31,8 & $26-37$ & & 74 & 25,2 & $20-30$ & & 28,3 & $25-32$ & \\
\hline Mulatos & 112 & 29,9 & $25-34$ & & 149 & 32,6 & $28-37$ & & 31,4 & $28-35$ & \\
\hline Negros & 16 & 23,9 & $13-34$ & & 24 & 37,5 & $26-49$ & & 30,5 & $23-38$ & \\
\hline Outros Mestiços & 4 & 16,7 & $8,7-31$ & & 12 & 23,1 & $13-34$ & & 21,1 & $12-30$ & \\
\hline
\end{tabular}

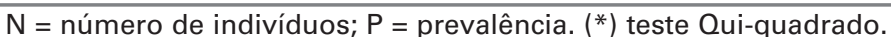

A síndrome metabólica foi identificada conforme critérios do National Cholesterol Education Program's - Adult Treatment Panel III (NCEP - ATP III) (3). 
Tabela 2. Estado nutricional de acordo com o índice de massa corpórea de mulheres com e sem síndrome metabólica, segundo classe socioeconômica.

\begin{tabular}{|c|c|c|c|c|c|c|c|c|}
\hline \multirow{2}{*}{$\begin{array}{l}\text { Classe Socioeconômica } \\
\text { Mulheres com SM }\end{array}$} & \multicolumn{2}{|c|}{$\mathbf{A}$} & \multicolumn{2}{|c|}{$\mathbf{B}$} & \multicolumn{2}{|c|}{ C } & \multicolumn{2}{|c|}{ D e E } \\
\hline & $\mathrm{N}$ & $\%$ & $\mathrm{~N}$ & $\%$ & $\mathrm{~N}$ & $\%$ & $\mathrm{~N}$ & $\%$ \\
\hline Baixo Peso & - & - & - & - & - & - & - & - \\
\hline Adequado & 1 & $(6,9)$ & 3 & $(6,0)$ & 9 & $(9,7)$ & 13 & $(11,9)$ \\
\hline Pré-obesidade & 11 & $(73,3)$ & 22 & $(44)$ & 32 & $(34,4)$ & 35 & $(32,1)$ \\
\hline Obesidade & 3 & $(20,0)$ & 25 & $(50)$ & 52 & $(55,9)$ & 60 & (56) \\
\hline \multicolumn{9}{|l|}{ Mulheres sem SM } \\
\hline Baixo Peso & 2 & $(2,9)$ & 8 & $(4,2)$ & 7 & $(3,7)$ & 7 & $(4,1)$ \\
\hline Adequado & 45 & $(66,2)$ & 105 & $(55,6)$ & 106 & $(56,4)$ & 92 & $(54,1)$ \\
\hline Pré-obesidade & 18 & $(26,5)$ & 57 & $(30,2)$ & 57 & $(30,2)$ & 56 & $(32,9)$ \\
\hline Obesidade & 3 & $(4,4)$ & 19 & (10.0) & 18 & $(9,6)$ & 15 & $(8,8)$ \\
\hline
\end{tabular}

$\mathrm{N}=$ número de indivíduos

Tabela 3. Indicadores bioquímicos, antropométricos e hemodinâmicos na amostra estudada, segundo sexo e presença de síndrome metabólica.

\begin{tabular}{|c|c|c|c|c|}
\hline \multirow[b]{2}{*}{ Indicadores } & \multicolumn{2}{|c|}{ Homens } & \multicolumn{2}{|c|}{ Mulheres } \\
\hline & sem Síndrome & com Síndrome & sem Síndrome & com Síndrome \\
\hline \multicolumn{5}{|l|}{ Bioquímicos } \\
\hline Triglicerídeos $(\log )^{* *}$ & $\begin{array}{c}N=521 \\
1,99 \pm 0,23^{*}\end{array}$ & $\begin{array}{c}N=217 \\
2,33 \pm 0,22 *\end{array}$ & $\begin{array}{c}N=614 \\
1,88 \pm 0,21 *\end{array}$ & $\begin{array}{c}N=265 \\
2,20 \pm 0,22 *\end{array}$ \\
\hline HDL-c (mg/dL) & $\begin{array}{c}N=520 \\
44,7 \pm 12,9 *\end{array}$ & $\begin{array}{c}N=202 \\
35,9 \pm 7,3^{*}\end{array}$ & $\begin{array}{c}N=617 \\
50,9 \pm 12,2^{*}\end{array}$ & $\begin{array}{c}N=257 \\
41,3 \pm 8,2^{*}\end{array}$ \\
\hline Glicemia (mg/dL) & $\begin{array}{c}N=499 \\
98,9 \pm 19,9 *\end{array}$ & $\begin{array}{c}N=211 \\
122,4 \pm 42,9 *\end{array}$ & $\begin{array}{c}N=599 \\
95,1 \pm 13,7^{*}\end{array}$ & $\begin{array}{c}N=261 \\
123,9 \pm 51,1^{*}\end{array}$ \\
\hline \multicolumn{5}{|l|}{ Antropométricos } \\
\hline IMC $\left(\mathrm{Kg} / \mathrm{m}^{2}\right)$ & $\begin{array}{c}N=524 \\
24,6 \pm 3,2^{*}\end{array}$ & $\begin{array}{c}N=216 \\
28,9 \pm 4,1^{*}\end{array}$ & $\begin{array}{c}N=615 \\
24,6 \pm 4,3^{*}\end{array}$ & $\begin{array}{c}N=267 \\
31,1 \pm 5,2^{*}\end{array}$ \\
\hline $\mathrm{RCO}$ & $\begin{array}{c}N=523 \\
0,90 \pm 0,06^{*}\end{array}$ & $\begin{array}{c}N=216 \\
0,96 \pm 0,06^{*}\end{array}$ & $\begin{array}{c}N=615 \\
0,80 \pm 0,06^{*}\end{array}$ & $\begin{array}{c}N=267 \\
0,89 \pm 0,07 *\end{array}$ \\
\hline Circunferência da cintura $(\mathrm{cm})$ & $\begin{array}{c}N=523 \\
85,6 \pm 8,9 *\end{array}$ & $\begin{array}{c}N=216 \\
97,6 \pm 11,2^{*}\end{array}$ & $\begin{array}{c}N=615 \\
78,5 \pm 10,2^{*}\end{array}$ & $\begin{array}{c}N=267 \\
95,3 \pm 10,6^{*}\end{array}$ \\
\hline \multicolumn{5}{|l|}{ Pressão Arterial } \\
\hline Sistólica (mmHg) & $\begin{array}{c}N=525 \\
125,9 \pm 17,8 *\end{array}$ & $\begin{array}{c}N=218 \\
139,2 \pm 19,6^{*}\end{array}$ & $\begin{array}{c}N=620 \\
119,0 \pm 19,6^{*}\end{array}$ & $\begin{array}{c}N=267 \\
142,0 \pm 23,8^{*}\end{array}$ \\
\hline Diastólica (mmHg) & $84,2 \pm 12,9 *$ & $93,0 \pm 13,3^{*}$ & $77,1 \pm 11,9 *$ & $91,8 \pm 13,5^{*}$ \\
\hline
\end{tabular}

Os valores são dados como média \pm desvio-padrão. $\mathrm{N}=$ número de indivíduos

Teste T de Student

$* \mathrm{P}<0,05$

* Transformado em valor logarítmico

rial, a hipertrigliceridemia, o baixo HDL-colesterol, o aumento da glicemia ou presença de diabetes e a presença de obesidade abdominal. Nas mulheres, a hipertensão também apareceu como o fator mais prevalente, seguida de baixo HDL-colesterol e presença de obesidade abdominal. Por último, diferente do que ocorre nos homens, apareceram a hipertrigliceridemia e a hiperglicemia ou presença de diabetes.

A figura 2 mostra o número de componentes para SM encontrado em mulheres e homens, em cada faixa etária. Pode-se observar que, na faixa etária de 25-34 anos, há grande predominância de indivíduos (em ambos os sexos) que são portadores de três componentes para SM $(70,9 \%$ no sexo feminino e 79,3 no masculino). Com o aumento da idade, observa-se um incremento no número de componentes, principalmente a partir dos 45 anos no sexo feminino.

\section{DISCUSSÃO}

A SM constitui uma entidade que tem sido objeto de crescente preocupação em todo o mundo, tendo em vista que os portadores da síndrome possuem achados clínicos e laboratoriais que são fortes preditores de agravos à saúde, particularmente à saúde cardiovascular. Apesar da existência de componentes bem definidos para o seu diagnóstico, a presença de fatores cau- 


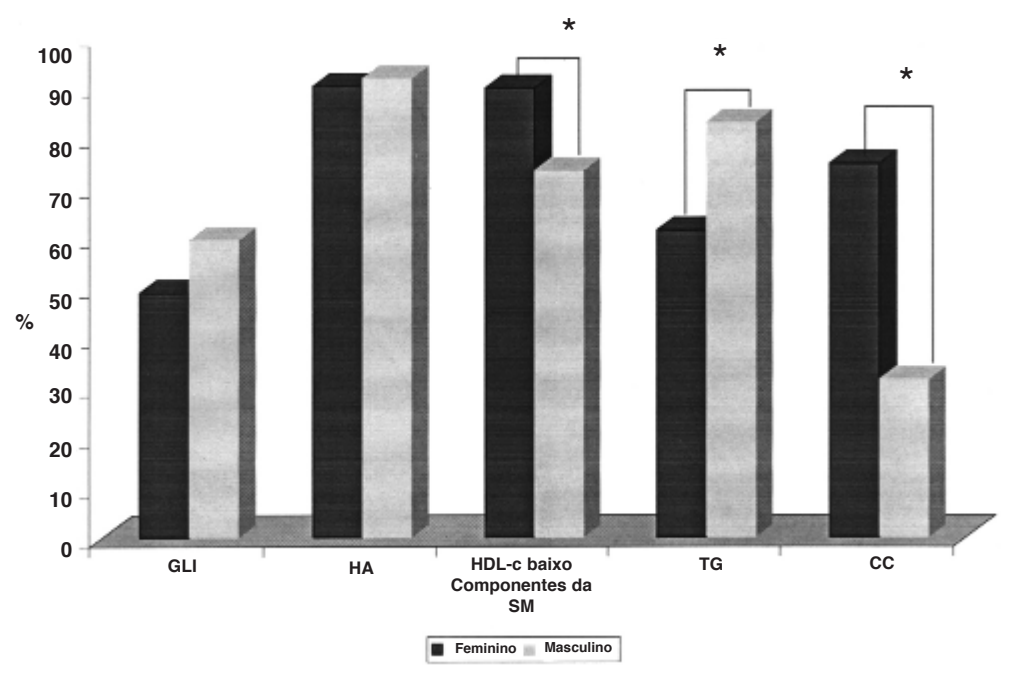

Figura 1. Distribuição dos componentes para Síndrome Metabólica de acordo com NCEP-ATP-III na população estudada. Vitória/ES - Brasil Test T

* $\mathrm{P}<0.05$ (diferença entre homens e mulheres com SM)

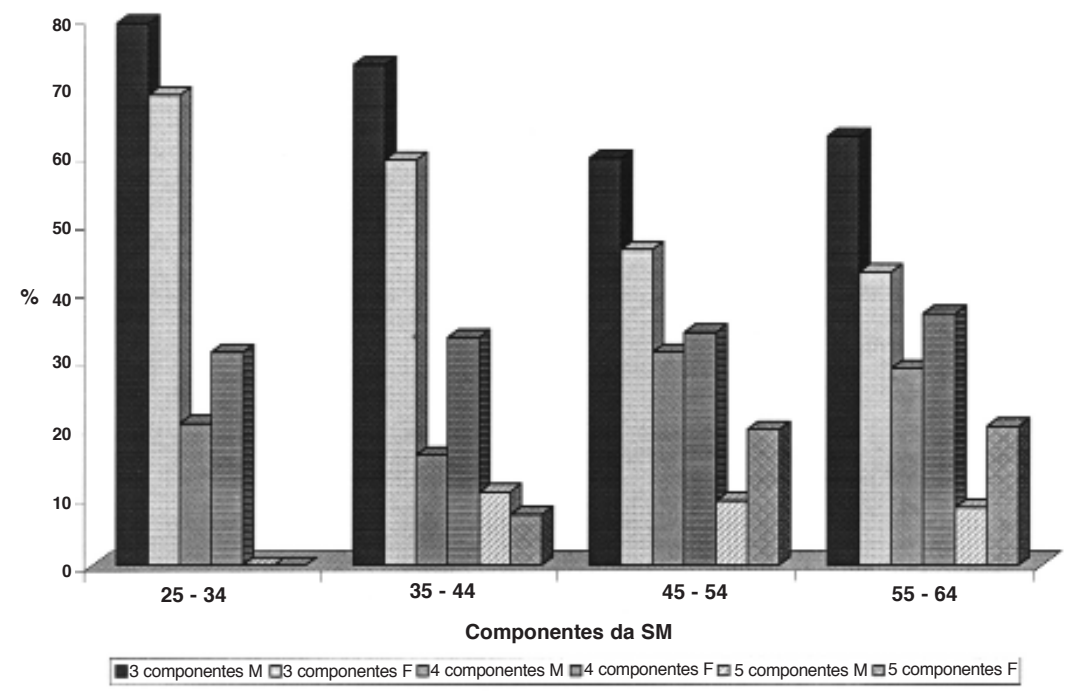

Figura 2. Distribuição dos componentes para síndrome metabólica em homens em Vitória/ES, 2000/01.

M: Masculino, F: Feminino

sais diversos para o desenvolvimento da SM torna a comparação de prevalência entre diferentes populações relativamente difícil, pois, em populações específicas, a apresentação da síndrome pode ocorrer com componentes individuais distintos. Diferenças na distribuição por sexo, faixa etária, dieta e grau de atividade física, além de características étnicas e genéticas, podem afetar o aparecimento dos diversos componentes da SM. Portanto, as prevalências descritas em nosso estudo são representativas de uma população brasileira urbana, heterogênea quanto à sua ascendência, constituída de adultos na faixa etária de 25-64 anos. Como a prevalência de alguns componentes da síndrome, notadamente a obesidade, tem sofrido 
alterações rápidas e significativas nas últimas décadas, o quadro da SM aqui traçado pode ter também se modificado, tendo em vista que a coleta de dados em campo foi feita em 2000 e 2001 . No entanto, como não há estudos representativos da população brasileira sobre SM, nossos achados constituem referência importante para outros estudos que venham a ser conduzidos em outros contextos econômicos e sociais. Além disso, torna-se referencial importante para a determinação da incidência da $S M$, o que também ainda não foi determinado na população brasileira em geral.

Deve-se ressaltar, no entanto, o estudo conduzido por Rosenbaum e cols. (17), também de base populacional, porém realizado exclusivamente em população nipo-brasileira. Nesse estudo, verificaram uma elevada prevalência de SM, utilizando tanto o critério recomendado pela OMS como o recomendado pelo NCEP $(55,4 \%$ e $47,4 \%$, respectivamente). Entretanto, houve diferença importante em relação à identificação de obesidade central como elemento constituinte da síndrome $(48,3 \%$ e $14,0 \%$, respectivamente). A utilização de componentes específicos para asiáticos, proposta pela OMS, aumentou o número de indivíduos com SM (58\%), e com conseqüente aumento de sua associação com as DCV (17).

Em estudos de prevalência, a representatividade da amostra constitui elemento crítico para a generalização dos resultados. A amostra usada em nosso estudo através da identificação domiciliar possui distribuição quanto ao sexo e faixa etária superponível aos dados coletados pelo censo de 2000 , podendo ser considerada, portanto, representativa da população de Vitória. Tendo em vista que a amostra foi planejada para cobrir proporcionalmente todo o espaço urbano da cidade, pode-se supor que as classes socioeconômicas também estejam equitativamente representadas na amostra. Em relação à amostra efetivamente estudada, observou-se pequeno viés de retorno, sendo que o comparecimento aos exames cresceu do extrato etário mais jovem (compareceram aos exames 71\% dos indivíduos de 25 a 34 anos) para o de maior idade (comparecimento de $81 \%$ dos indivíduos de 55 a 64 anos). Dessa forma, a média de idade da amostra estudada foi cerca de 2 anos maior do que a da amostra domiciliar. Portanto, as estimativas da prevalência populacional da SM na população em estudo, após levar em consideração esse pequeno viés, situou-se em $29,8 \%$ (IC 95\% 28-32\%). Este valor foi similar aos $30,0 \%$ encontrados por Oliveira e cols. (18) em amostra aleatória de 240 moradores (25-87 anos) do distrito de Cavunge, no semi-árido da Bahia. Em que pesem as diferenças de faixa etária, esses dados indicam que a SM apresenta, hoje, elevada prevalência na população brasileira, tanto residentes em áreas urbanas como rurais.

Nas últimas décadas, a população brasileira convive com um incremento na mortalidade por doenças crônicas não transmissíveis (DCNT), sendo que, no patamar superior, encontram-se as doenças cardiovasculares, em particular as doenças do aparelho circulatório. Essa posição de destaque é encontrada em todas as regiões do país (13). Estudo publicado em 2004, que teve por objetivo descrever os diferenciais existentes quanto ao padrão epidemiológico no Brasil e grandes regiões, utilizando o indicador DALY (Disability Ajusted Life of Years), mostrou que as DCNT responderam por $66,3 \%$ da carga de doença no Brasil (19).

Segundo a Organização Mundial de Saúde (OMS), o aumento da incidência das DCNT pode ser fortemente atribuído ao sedentarismo, juntamente com a adoção de dietas desequilibradas, as quais se popularizaram em todo o mundo, incluindo o Brasil, a partir da segunda metade do século XX (20). Este cenário não é exclusivo dos países desenvolvidos, já que o perfil de morbimortalidade do Brasil é indicativo de que os hábitos alimentares e estilo de vida da população vêm colaborando fortemente para as mudanças no perfil epidemiológico. Desta forma, a SM ganha importância por ser considerada um transtorno complexo representado por um conjunto de fatores usualmente relacionado à deposição central de gordura e à resistência à insulina, contribuindo, assim, com o aumento do risco de desenvolvimento das SCD e diabetes (3).

Vários estudos mostram a elevada prevalência de SM em diferentes populações. $\mathrm{Na}$ população americana, a prevalência de SM na faixa etária de 20 a 69 anos foi de 23,7\%, sendo que, entre 20 a 29 anos, a prevalência foi de 6,7\%, aumentando de forma progressiva até $43,5 \%$ na faixa etária de 60 a 69 anos. Os resultados indicaram que não há diferença em relação à prevalência de SM entre os sexos, de acordo com as diferentes faixas etárias (21), resultado similar ao encontrado em Vitória. Constatamos em nosso estudo, entretanto, prevalência maior da SM nos extratos etários mais jovens, fator este que deve ser objeto de preocupação adicional, pois o mesmo indica agravamento nas próximas décadas das epidemias de diabetes, hipertensão arterial e eventos cardiovasculares fatais e não-fatais, o que certamente exigirá investimentos crescentes nessa área de atenção à saúde.

Estudo realizado na Turquia, também utilizando componentes do NCEP ATP-III, mostrou que a prevalência de SM foi elevada entre indivíduos de 
30-39 anos de áreas urbanas, situando-se em torno de $20 \%$ (22). Já na população urbana da Coréia, considerando-se a faixa etária de 30-80 anos e o mesmo critério, foi identificada uma prevalência de $16 \% \mathrm{em}$ homens e 10,7\% em mulheres. Nesse mesmo estudo, ao se utilizar componentes nos quais os valores da circunferência da cintura foram reduzidos de $102 \mathrm{~cm}$ para $90 \mathrm{~cm}$ em homens e de $88 \mathrm{~cm}$ para $80 \mathrm{~cm}$ para as mulheres, a prevalência de SM aumentou para $29 \%$ e $16,8 \%$, respectivamente $(23)$, à semelhança do ocorrido no estudo com nipo-brasileiros (17).

$\mathrm{Na}$ população mexicana, a prevalência de SM variou de acordo com o critério utilizado. Ao adotar o critério para diagnóstico da OMS, a prevalência total de SM foi de $13,6 \%$, ao passo que ao utilizar o critério do NCEP ATP III, a prevalência de SM totalizou $26,6 \%(24)$.

Alguns estudos sugeriram existir associação entre a condição socioeconômica e o aparecimento da SM. Estudo realizado na França, com o objetivo de verificar a relação entre renda familiar e SM em indivíduos de ambos os sexos, mostrou que a renda familiar se associou inversamente com a SM em mulheres, mas não em homens (25). Achado similar foi observado em nosso estudo, onde a prevalência da SM em mulheres da classe A foi de $17,9 \%$ e no grupo de menor renda (classes D e E) alcançou o valor de $38,7 \%$. A renda é fator que tem impacto nas práticas de lazer, nos níveis de atividade física, no acesso a uma alimentação mais saudável etc. Esses fatores sabidamente são subjacentes ao desenvolvimento da SM. Portanto, nossos dados sugerem que uma renda familiar mais baixa tem maior impacto no sexo feminino, dificultado a manutenção de hábitos de vida saudável e de outras estratégias úteis no controle do peso corporal. Uma possível explicação acerca da diferença entre os sexos pode estar relacionada ao tipo de trabalho exercido pelos homens em classes socioeconômicas menos favorecidas, com ocupações que demandam mais esforço físico e conseqüentemente maior gasto energético e menor tendência ao acúmulo de gordura central. Outra explicação poderia ser a diferença entre sexos nas formas de lazer, onde os homens teriam acesso maior a atividades que facilitariam o controle do peso, ao contrário do que ocorreria com as mulheres de menor renda. Entretanto, as razões das diferenças no desenvolvimento da SM entre os sexos em relação ao gradiente socioeconômico ainda requerem estudos adicionais que demandam o conhecimento de como variam os componentes individuais da SM nas diferentes classes sociais. Estudos paralelos realizados em nossa amostra evidenciam que, nas classes socioeconômicas mais baixas, é maior o consumo de sal e a prevalência de hipertensão arterial (14), assim como é maior a prevalência de obesidade entre as mulheres.

Determinantes sociais são firmemente correlacionados com a SM, porém a contribuição desses não está totalmente elucidada. Esses, por sua vez, influenciam hábitos alimentares, prática de atividade física e hábitos de vida deletérios, o que pode contribuir para o desfecho e aglomeração de doenças crônicas (26). Este fato pode ser explicado pelos elevados índices de pré-obesidade e obesidade encontrados nas mulheres das classes C e D, corroborando com a hipótese de a obesidade ser o fator desencadeante da SM nesta população.

A última Pesquisa de Orçamento Familiar confirmou que, além do incremento do sobrepeso e da obesidade, houve mudanças expressivas nos hábitos alimentares dos brasileiros nas últimas três décadas. Neste período, o brasileiro diversificou a sua alimentação, houve o incremento na aquisição de produtos industrializados e redução de alimentos in natura, como frutas e hortaliças. Essa pesquisa também confirmou que o brasileiro vem reduzindo o consumo de gêneros alimentícios tradicionais como arroz e o feijão $(9)$.

Observa-se, ainda, em ambos os sexos, que a hipertensão arterial apareceu com maior freqüência nos indivíduos com SM dentre os componentes analisados, evidenciando a importante contribuição deste fator para o desfecho estudado. Considerada uma das doenças mais prevalentes na população brasileira e na maioria dos países emergentes, a hipertensão também tem sido considerada a morbidade mais comum na população adulta. Está diretamente associada com a alta mortalidade cardiovascular, e suas conseqüências representam elevada freqüência nos serviços de emergência no Brasil (27) e um desafio para a atenção primária à saúde (28).

Estima-se que a prevalência de hipertensão na população adulta brasileira situa-se entre $25 \%$ a $30 \%$ (29), contribuindo de forma crescente com a mortalidade cardiovascular (6). O estudo de Vitória indicou prevalência de 38\% (30), atingindo 43,6\% nos homens de 25 a 64 anos de idade. Nesse mesmo estudo, observou-se que as mulheres de classes socioeconômicas mais elevadas apresentaram menor prevalência de hipertensão e melhores indicadores nutricionais.

No presente estudo, é notório o grande percentual de indivíduos apresentando valores elevados da circunferência da cintura, principalmente no sexo feminino. É importante ressaltar a íntima relação entre 
obesidade central e o surgimento da SM (31). Na coorte do Insulin Resistance Atheresclerosis Study IRAS (32), que teve por objetivo verificar a incidência de SM em indivíduos adultos não-diabéticos, notou-se que a circunferência abdominal foi a variável com maior poder de predição para o desenvolvimento da SM.

Em nosso estudo, o segundo critério para SM mais freqüente entre as mulheres foi o baixo HDL-c e, entre os homens, a hipertrigliceridemia. Alterações do metabolismo dos lipídeos em indivíduos com resistência à insulina aumentam a importância do papel da insulina no metabolismo dos ácidos graxos livres e na produção de triglicérides ricos em VLDL (32). Esse quadro é altamente aterogênico e causa, por isso, aumento no risco de doença cardiovascular nos indivíduos com resistência à insulina aumentada (7).

Diferentes estudos têm associado SM com doença cardiovascular. Um estudo, ao avaliar mulheres americanas com idade igual ou maior que 45 anos, demonstrou que aquelas com infarto agudo do miocárdio não fatal tinham 4,7 vezes mais chances de apresentar três ou mais componentes da síndrome, quando comparadas com as controles pareadas por idade, grupo racial e tabagismo (33)

É bem documentado que a idade contribui para o aparecimento de SM e com o acúmulo de desordens metabólicas. Ao analisar o número de componentes por sexo e faixa etária, observa-se um incremento no número de componentes por faixa etária em ambos os sexos, com maior representatividade no sexo feminino. Um estudo, ao avaliar o risco relativo de desenvolver doença cardiovascular e diabetes, relacionado ao número de componentes para SM, concluiu que o risco de desenvolver doença cardiovascular é seis vezes maior quando quatro ou mais componentes estavam presentes (34).

Este estudo mostrou que a prevalência de SM encontrada na cidade de Vitória é elevada, com grande contribuição da hipertensão arterial para o seu diagnóstico em ambos os sexos. Observa-se, também, uma elevada prevalência dessa síndrome em indivíduos mais jovens, o que pode estar contribuindo para a mortalidade em idades ainda produtivas, ou ainda com aposentadorias provenientes de invalidez. O estudo sugere que ações específicas devem ser realizadas para o controle dos fatores que determinam o desenvolvimento da SM, notadamente entre os mais jovens. A promoção da saúde deve contemplar aspectos relacionados à atividade física e hábitos saudáveis de alimentação, atuando de forma preventiva, o que poderá contribuir para a redução da mortalidade cardiovascular.

\section{AGRADECIMENTOS}

Ao CNPq (processo no 521148/97/6) e ao Fundo de Apoio à Ciência e Tecnologia — Facitec/Prefeitura de Vitória.

\section{REFERÊNCIAS}

1. Mendonça $\mathrm{CP}$, Anjos LA. Aspectos das práticas alimentares e da atividade física como determinantes do crescimento do sobrepeso/obesidade no Brasil. Cad Saúde Pública 2004;20(3):698-709.

2. Pozzan R, Pozzan R, Magalhães MEC, Brandão AA, Brandão AP. Dislipidemia, síndrome metabólica e risco cardiovascular. RSOCERJ 2004;17(2):97-104.

3. Sociedade Brasileira de Hipertensão. I Diretriz Brasileira de Diagnóstico e Tratamento da Síndrome Metabólica. Hipertensão 2004;7(4).

4. Miname MH, Chacra APM. Síndrome metabólica. Rev Soc Cardiol 2005;15(6):482-9.

5. Reaven G. Role of insulin resistance in human disease. Diabetes 1988;37:1595-607.

6. Lotufo PA. Mortalidade precoce por doenças do coração no Brasil. Comparação com outros países. Arq Bras Cardiol 1998;70:321-5.

7. Lakka HM, Lakka TA, Tuomilehto J, Sivenius J, Salonen JT. The metabolic syndrome and total and cardiovascular disease mortal in middle-aged men. JAMA 2002;288(21):2709-16.

8. Ministério da Saúde. Pesquisa nacional sobre saúde e nutrição. Brasília: INAN, 1990.

9. IBGE. Pesquisa de orçamentos familiares. Análise da disponibilidade domiciliar de alimentos e do estado nutricional no Brasil. Rio de Janeiro: IBGE, 2004.

10. Oliveira CL, Mello MT, Cintra IP. Obesidade e síndrome metabólica na infância e adolescência. Rev Nutr 2004; 17(2):237-45.

11. Gorter PM, Olijhoek JK, Van Der Graaf Y, Algra A, Rabelink TJ, Visseren FL. Prevalence of the metabolic syndrome in pacients with coronary heart disease, cerebrovascular disease, peripheral arterial disease or abdominal aneurysm. Atherosclerosis 2004;173(2):361-7.

12. Chor D, Fonseca MJN, Andrade CR, Waissman W, Lotufo PA. Doenças cardiovasculares: panorama da mortalidade no Brasil In: Minayo MCN (org). Os muitos Brasis - saúde e população na década de $\mathbf{8 0}$. São Paulo: HUCITEC ABRASÇO, 1995. pp. 57-86.

13. Ministério da Saúde. Saúde Brasil 2004: uma análise da situação de saúde. Secretaria de Vigilância em Saúde, Departamento de Análise de Situação de Saúde. Brasília: Ministério da saúde, 2004. p. 350.

14 Molina MCB, Cunha RS, Herkenhoff FL, Mill JG. Hipertensão arterial e consumo de sal em região urbana. Rev Saúde Pública 2003:37(6):743-50.

15. Whorld Health Organization. Obesity: preventing and managing the global epidemic. Report of a WHO Consultation. WHO Technical Report Series 894. Geneva: World Health Organization, 2000

16. Fundação Instituto Brasileiro de Geografia e Estatística FIBGE. Pesquisa por amostragem de domicílios 1996: microdados. FIBGE: Rio de Janeiro, 1997.

17. Rosenbaum P, Gimeno SGA, Sanudo A, Franco LJ, Ferreira SLG. Analysis of criteria for metabolic syndrome in a population-based study of Japanese-Brazilians. Diabetes Obes Metab 2005;7(4):352-9.

18. Oliveira EP, Souza MLA, Lima MDA. Prevalência de síndrome metabólica em uma área rural do semi-árido baiano. Arq Bras Endocrinol Metab 2006;80:456-65.

19. Schramm JMA, Oliveira AF, Leite IC, Valente JG, Cadelha AMJ, Portela MC, et al. Transição demográfica e o estudo de carga da doença no Brasil. Ciênc Saúde Colet 2004; 9(4):897-908. 
20. Whorld Health Organization. Technical Report Series 916. Diet, nutrition and prevention of chronic diseases. Genebra: WHO, 2003

21. Ford ES, Giles WH, Dietz WH. Prevalence of the metabolic syndrome among US adults: findings from the third National Health and Nutrition Examination Survey. JAMA 2002;287:356-9.

22. Soysal A, Demiral Y, Soysal D, Uçku R, Koseoglu M, Aksakoglu G. The prevalence of metabolic syndrome among young adults in Izmir Turkey. Anadolu Kardiyol Derg 2005;5(3):196-201.

23. Oh J-Y HYS, Sung Y-A CBE. Prevalence and actor analysis of metabolic syndrome in an urban Korean population. Diabetes Care 2004;27:2027-32.

24. Aguilar-Salinas CA, Rojas R, Gómes Perez FJ, Valles V, RiosTorres JM, Franco A. High prevalence of metabolic syndrome in Mexico. Arch Med Res 2004;35(1):76-81.

25. Dallongeville J, Cottel D, Ferrières J, Arveiler D, Bingham A, Ruidavets JB, et al. Household Income is associated with the risk of metabolic syndrome in a sex-specific manner. Diabetes Care 2005;28(2):409-15.

26. Ecob R, Smith GD. Income and health: What is the nature of relation ship? Soc Sci Med 1999;48:693-705.

27. Lessa I. Epidemiologia da hipertensão arterial sistêmica e da insuficiência cardíaca no Brasil, 2001. Rev Bras Hipertens 2001;8(4):383-92.

28. Lessa I. Doenças crônicas não transmissíveis no Brasil: um desafio para a complexa tarefa de vigilância. Ciênc Saúde Colet 2004;4(9):931-43.

29. Lessa I. Os programas nacionais de educação de educação e controle para DCNT. In: Lessa I (org). Adulto brasileiro e as doenças da mortalidade. Epidemiologia das doenças não transmissíveis no Brasil. São Paulo: HUCITEC, 1998. pp. 241-50.
30. Mill JG, Molina MDC, Silva IO, Marquezine AL, Ferreira AVL, Cunha RS, et al. Epidemiologia da hipertensão arterial na cidade de Vitória, Espírito Santo. Hipertensão 2004;7(3):109-16.

31. Matos AFG, Moreira RO, Guedes, EP. Aspectos neuroendócrinos da Síndrome Metabólica. Arq Bras Endocrinol Metab 2003;47(4):410-21.

32. Palaniappan L, Carnethon MR, Wang Y, Hanley AJG, Fortmann SP, Haffner SM, et al. Predictors of the incident metabolic syndrome in adults: the Insulin Resistance Atherosclerosis Study. Diabetes Care 2004;27(3):788-93.

33. Amowitz LL, Ridker PM, Rifai N, Loughrey CM, Komaroff AL. High prevalence of metabolic syndrome among young women with nonfatal myocardial infarction. J Women Health 2004; 13:165-75.

34. Klein BE, Klein R, Lee KE. Components of the metabolic syndrome and risk of cardiovascular disease and diabetes in Beaver Dam. Diabetes Care 2002;25:1790-4.

\section{Endereço para correspondência:}

Maria del Carmen Bisi Molina

Programa de Pós-Graduação em Atenção à Saúde Coletiva

Centro de Ciências da Saúde - CCS/UFES

Av. Marechal Campos 1468

29040-090 Vitória, ES

E-mail: mdcarmen@npd.ufes.br / mdmolina@uol.com.br 